\title{
Flucht aus der DDR. Inszienierungen am Beispiel von Julia Francks Lagerfeuer und dessen Verfilmung Westen
}

\begin{abstract}
Anikó Zsigmond
Fleeing the GDR. Images of flight in Julia Franck's Lagerfeuer and its film adaptation Westen

Abstract

The paper deals with the topic of flight from the GDR to the GFR in the 70s, as shown in Julia Franck's novel Lagerfeuer and its film adaptation Westen. The novel is partially based on the author's own experience as a child when her family left the GDR. However, the novel is more like a fictitious story of a scholarly person who leaves the communist country. The film adaptation depicts the hopeless waiting in a refugee camp near Berlin from four perspectives. Accordingly, four ways of life are presented along with the political background, personal motivations and crises. The paper focuses on questions of foreignness and freedom that the protagonists have to experience and cope with in order to survive. The motives of fear and persecution are examined in scenes like the interrogation by the authorities. Last but not least, the paper also discusses the differences between the literary text and its film adaptation.
\end{abstract}

Keywords: flight from GDR; freedom; fear; persecution; literary text and film adaptation

Schlüsselwörter: Flucht aus der DDR; Freiheit; Angst; Verfolgung; literarischer Text und Verfilmung

Subject-Affiliation in New CEEOL: Language and Literature - Studies of Literature - German Literature

DOI: $10.36007 /$ eruedu.2020.3.094-102

\section{Einleitung: der politische und biographische Hintergrund}

Julia Franck hat mit Lagerfeuer einen deutsch-deutschen Flüchtlingsroman geschrieben und die Geschichte ihrer Kindheit aufgearbeitet. (Schmelcher 2006) Das geht aus einem Interview mit der Autorin hervor. Sie nahm nämlich den gleichen Weg wie ihre Protagonistin. Autobiographisches und Fiktionales stimmen in vielen Details überein. 1978 reiste Julia Franck aus der DDR aus und lebte acht Monate im West-Berliner Notaufnahmelager Marienfelde, wo auch Lagerfeuer spielt. Marienfelde ist eine historische Realität. In den 1970er Jahren war es mit Flüchtlingen aus der DDR überfüllt. Im oben erwähnten Interview bezeichnet die Autorin das Lager als einen Lebensbruch, denn hier nahmen Schicksale einen 
Wendepunkt, hier wurden soziale und lokale Beziehungen abgebrochen und auch eine andere Weltsicht machte sich kund. Franck zufolge erlebte man, dass „der ehemalige Feind [...] plötzlich selbst war" (Schmelcher 2006), was große Unsicherheit hervorrief und die frühere Vorstellung von Opfern und Tätern völlig ins Wanken brachte. Marienfelde war also ein Krisenpunkt, zu dem die Flüchtlinge kamen, wobei sie selbst aus einer Krise geflohen waren und in eine unsichere Zukunft gelangten. Lagerfeuer ist ein deutscher Flüchtlingsroman. Das traurige Thema der Flucht aus der DDR fand allerdings in der deutschen Literatur wenig Beachtung, denn in den Jahren nach dem Mauerbau ging die Zahl der binnendeutschen Flüchtlinge zurück und in Marienfelde waren es in den 70er Jahren 4000 Geflüchtete, zu wenig, um das Thema literarisch auf diese Art zu verarbeiten. (Schmelcher 2006) Es gibt eher satirische Zugänge zur DDR-Vergangenheit wie Thomas Brussigs Helden wie wir oder den Film Good bye Lenin von Wolfgang Becker, aber Franck geht nicht diesen Weg. Ihr sind die Vielstimmigkeit, und die vielen Perspektiven auf die deutsche Geschichte wichtig.

\section{Geschichte und Struktur}

Der Roman Lagerfeuer verbindet vier Erwachsenenschicksale im Notaufnahmelager miteinander: Die aus einer halbjüdischen Familie kommende Nelly Senff reist mit ihrem Sohn Aleksej und ihrer Tochter Katja aus der DDR aus, denn nach dem unaufgeklärten Tod des Vaters ihrer Kinder fühlt sie sich in der DDR nicht mehr wohl. Die aus Polen stammende Krystina hat deutsche Vorfahren und will ihren krebskranken Bruder in der BRD operieren lassen. Der amerikanische Geheimdienstmann John Bird führt Verhöre mit den Flüchtlingen, und der Schauspieler Hans Pischke wurde aus einem DDR-Gefängnis freigekauft. In der narrativen Struktur unterscheiden wir vier Erzähler, die aus der Ich-Perspektive erzählen. Die wichtigste Erzählerstimme ist die von Nelly, die voll von Erfahrungen, wie Wünschen und Ängsten ist. Sie reist nach West-Berlin, weil sie von einem besseren Leben träumt, aber sie muss bis zur Ausreise, bei der Ausreise und nach der Ausreise immer wieder Erniedrigungen erfahren. Sie möchte in eine freie Welt, aber sie ist unerträglich vielen Befragungen ausgesetzt, so dass sie langsam ihre Träume und Hoffnungen aufgibt und sich mit einer Aussichtslosigkeit abgefunden sieht. Aus dem Notaufnahmelager führt kein Ausweg mehr, nachts herrscht ein Ausgehverbot, die Flüchtlinge wohnen beengt, die Kinder werden in der Schule als Fremde verspottet oder sogar von anderen westdeutschen Kindern misshandelt. Potenzielle Hilfe wird innen nicht angeboten, die Personen sind auf Wohltätigkeit angewiesen, Arbeits- und Neuanfangsmöglichkeiten gibt es kaum. Statt der Freiheit erleben sie die Enge, die reale Grenze der Mauer wird duch die mentale Grenze der Exlusion abgelöst. Das ist schockierend und desillusionierend. Die erzählten Geschichten begleitet eine bedrückende und aussichtslose Atmosphäre. 


\section{Motivstruktur}

Der Beitrag konzertriert sich auf die Perspektive von Nelly Senff, umso mehr, weil im Film ebenfalls fast ausschließlich ihre Geschichte im Fokus steht. Die anderen drei Erzähler erscheinen eher als Nebenfiguren und unter dem Aspekt ihrer Beziehung zu Nelly.

Daher werden folgende Motive in oppositionellen Motivkomplexen erläutert: Freiheit, Grenze, Begrenztheit, Gefangenschaft und Ausgrenzung, Angst, Bedrohung, Hoffnung und Enttäuschung, und schließlich die Befragungen. Nelly Senff befindet sich in einer Situation voller existenzieller und emotionaler Widersprüche, die der politischen Atmosphäre entspringen. Die reale Erfahrung, nachdrücklich politisch bedroht zu sein, macht das individuelle Leben in der DDR unsicher, aber die Flucht vor dieser Gefahr bringt ebenso große Unsicherheiten, sogar Aussichtslosigkeit mit sich. Der größte Unterschied zwischen dem Buch und der Verfilmung liegt eben in dem Abschluss und in der Überwindung dieser Widersprüche. Die Geschichte des Romans bleibt in einem Zustand der Verharrung stecken. Aus dem Notaufnahmelager Marienfeld entkommt man nicht, hier landen und verharren die Flüchtlinge. Der Abschluss des Buches ist deprimierend. Die Hoffnungen gehen verloren. Der Abschluss der Verfilmung ist jedoch eine andere Perspektive, wobei der vorübergehende Zustand der Verharrung überwunden und aufgehoben wird. Nelly mietet eine Wohnung und bekommt einen Job.

\subsection{Motivkomplex Freiheit, Grenze, Begrenztheit, Ausgrenzung, Gefan- genschaft}

Das Buch beginnt mit dem Kapitel Nelly Senff fährt über eine Brücke. Es handelt sich um die Bornholmer Brücke, über die Nelly mit ihren zwei Kindern nach West-Berlin unter dem Vorwand zu heiraten fährt. Die Brücke ist ein wichtiges Symbol der Grenze und spielt in der politischen Geschichte der DDR eine wichtige Rolle. Sie war ein wichtiger Grenzübergang, einer der Orte, an denen sich auch der Mauerfall 1989 vollzog. Bis 1989 markierte diese Brücke die Trennung, danach die Einigung. Für die Protagonistin bedeutet die Brücke die Grenze, den Übergang und den Weg zur Freiheit. Die räumliche Kürze des Weges steht im Kontrast zur zeitlichen Länge des Übergangs. Ein Weg, den man normalerweise binnen zwei Minuten zurücklegen könnte, dauert stundenlang in der Nacht der endgültigen Ausreise. Die Dunkelheit der Dämmerung kontrastiert mit den Lampen, die die Grenzübergänger beleuchten.

„Über dem dunklen Fenster brannte eine Neonlampe. Ich wartete, daß ein Licht anging, aber das Fenster blieb schwarz. [...] Aber die Scheiben dieses Fensters hier waren schwarz, besonders schwarz, tiefschwarz, kohlschwarz, rabenschwarz, je länger ich hinübersah, desto unnatürlicher schien es mir. Alles Licht aufgesogen." (Frank 2003, 10) 
Die Steigerung von schwarz verdeutlicht die Aussichtslosigkeit und Angst. Es geht um das Fenster eines Flachbaus, in den die Reisenden gebracht werden, um durchsucht und befragt zu werden. Dieser Bau symbolisiert die Macht der Diktatur, die in den Ausreisenden Angst entstehen lässt. Schwarz verschluckt das Licht, so wie die Macht ihre Bürger verschluckt.

Folgende Sonnenmetapher macht diese Überganssituation deutlich:

„Bis die Sonne uns voran zwischen den Häusern versank und noch einmal in der Fensterscheibe des Wachturms hoch über uns aufleuchtete, als wollte sie uns locken und versprechen, daß wir sie schon morgen wiedersähen, im Westen, wenn wir ihr nur folgten, und weg war sie und ließ uns hier in der Dämmerung mit ein paar Feuerstreifen am Himmel stehen und die Schatten schluckten nicht nur uns, sondern die ganze Stadt in unserem Rücken [...]" (Frank 2003, 8)

Typische Requisiten des Grenzübergangs sind außerdem der Wachturm und die Uniform. Überhaupt steht Nelly in West-Berlin fast ausschließlich mit Lagerbewohnern oder Angehörigen von Behörden in Kontakt: „[...] der ganze Raum könnte voll sein von Staatsdienern in Uniform, Volkspolizisten, Angehörigen der Staatssicherheit, Grenzsoldaten, Oberen, Lehrlingen, Helfern - aber dann verlöre der einzelne an Autorität” (Frank 2003, 11).

Dieses Gefühl der Einsamkeit, Verlassenheit, des Eingeschlossen-Seins und der Machtlosigkeit verfolgt die Hauptfigur in beiden Räumen: im Osten und im Westen. Sie erlebt überall eine Grenze, erstmal die physische, dann die mentale. Sie kann über ihre Möglichkeiten nicht hinausgehen. Die Wege und die Zugänge sind versperrt. Sie bekommt nicht einmal ein Visum für die DDR, um ihre Mutter und Geschwister besuchen zu dürfen. Sie ist zwischen den Räumen steckengeblieben.

\subsection{Motivkomplex Angst, Bedrohung und Hoffnung}

Die Angst verfolgt die Hauptfigur die ganze Geschichte lang. Sie ist zwar eine starke Persönlichkeit, unter der politischen Bedrohung und der eigenen Machtlosigkeit leidet sie aber. Beim Grenzübergang wird sie von einer körperlichen Schwäche erfasst: „Schlecht war jetzt mir [...]. Erst jetzt bemerkte ich mein Zittern” (Frank 2003,10). Einerseits reagiert sie so stark, weil sie um ihre Kinder große Angst hat, andererseits davor, dass sie sie nicht genug schützen kann. Hilflos war sie schon beim Umgang der Fremden mit ihren Kindern, nachdem sie die Ausreiseabsicht den Bekannten erzählt hat, so zum Beispiel als die Lehrerin der kleinen Tochter einredet, dass sie nun Kapitalisten und Feinde würden und das schlecht sei. Am Grenzübergang müssen die Kinder getrennt ins Gebäude zur Befragung gehen. Nelly erlebt dabei die größten Leiden, fast einen Verfolgungswahn. Der beruht aber auf den realen Mechanismen der kommunistischen Diktatur. Das Bild des unterirdischen Labyrinths suggeriert Angst, Ohnmacht und Verlassenheit, also den existentiellen Verlust und die Entfremdung.

„Die Tür blieb so verschlossen, daß ich überlegte, ob ich mich getäuscht hatte und meine Kinder in einer ganz anderen Baracke verschwunden wären. [...] Vielleicht 
führte ein unterirdischer Gang in ein entlegenes Polizeilager, dierkt ins Zentralkomitee, in die dunklen Gewölbe der Staatssicherheit. [...] Womöglich befand sich unter dem Schloßplatz ein weitverzweigtes Labyrinth mit speziellen Verliesen, in denen Kinder der Flüchtlinge eingesperrt und zur Besserung genötigt wurden. Bis sie bereit waren, von staatstreuen Bürgern in sozialistische Familien aufgenommen zu werden. [...] Was zählte schon das Papier, die Genehmigung, wenn sie mich einfach verschwinden ließen, ganz und gar, und die Kinder in ein Heim steckten? Zwansadoption. Darüber gab es Gerüchte. Insbesondere Feinde des Landes, aber auch Feinde der sozialistischen Demokratie." (Frank 2003, 12)

Auch im Westen, im Notaufnahmelager kann Nelly ihre Ängste und Ohnmacht nicht loswerden. Infolge der ständigen Verhöre ist sie misstraurisch und zweifelt an der Ehrlichkeit vorgespielter Hilfsbereitschaft. Das geht aus einer Szene mit Doktor Rothe hervor. Die Hauptfigur versucht der Hilfeleistung stolz und entschlossen zu entgehen. Sie fragt: „Was für eine Hilfe soll es sein? Wobei?” (Frank 2003, 235). Doktor Rothe versucht ihr einzureden, dass sie in einer schrecklichen und unwürdigen, sogar auswegslosen Situation mit den Kindern lebe. Das widerlegt Nelly, indem sie behauptet, es sei eine ungewohnte Situation, aber sie sei aus freier Entscheidung hergekommen. Sie würde einen Scheck bekommen und man würde ihr bei der Suche nach einer Wohnung helfen. Nelly lehnt misstraurisch diese Hilfe ab, aber der Mann versucht sie zu überzeugen: „Glauben Sie nicht, daß ihre Kinder es als Demütigung erleben, durch eine Schranke nach Hause und wieder hinaus ins Freie zu treten?” (Frank 2003, 237). Der Mann meint, Nelly könne diese Situation mit ihrer Weigerung nicht lösen und sie sei wie viele „Opfer menschenfeindlicher und unwürdiger Systeme” (Frank 2003, 243).

Nelly hat ihre Träume auch aufgegeben. Sie hat ihren Geliebten, den Vater ihrer Kinder, einen russischen Wissenschaftler auf ungeklärte Weise verloren. Er ist in West-Berlin spurlos verschwunden, und so ist das Leben für Nelly in der DDR noch unerträglicher geworden. Überall vermutet man, dass der Mann ein Agent war. Sie hat schon längst den Glauben daran aufgegeben, dass Wassilij je zurückkehrt. Der Traum im Allgemeinen ist ein Ausweg. Das erklärt sie dem westdeutschen Agenten, der die Verhöre leitet: „[...] wissen Sie, man tröstet sich wohl damit, man erzeugt im Traum diese Fata Morgana, den Ort, aber auch die Zeit, die eine andere ist. [...] Als befinde man sich im gemeinsamen Koordinatensystem irdischer Himmelsrichtungen" (Frank 2003, 96).

In der DDR kann man den Anderen nicht vertrauen, in jedem Menschen steckt ein potenzieller Mörder, der ein Doppelleben führt, mal ein Vater, mal ein Offizier des Geheimdienstes zu sein. Dieses Leben und diese Unsicherheit will sie im Westen loswerden.

\subsection{Motivkomplex Verhöre und Befragungen}

Die Hauptfigur und ihre Kinder sind von der Einreichunng des Ausreiseantrags an ständigen Befragungen und Verhören ausgesetzt. Die Kinder erleben das in der Schule, was besonders auf das kleinere Kind, das Mädchen einen großen Einfluss 
hat. Ihr wird dermaßen Angst eingejagt, dass sie nicht einmal schlafen kann. Bezeichnungen wie Feind der Heimat zu sein verunsichern sie. Im ersten Kapitel, als sie die Grenze passieren, werden die Kinder von Nelly getrennt und verhört. Nelly, die Ich-Erzählerin, erwägt in einem inneren Monolog, welche Fragen die Kinder bekommen werden und wie mit innen umgegangen wird.

„Sie werden Katja und Aleksej fragen, warum wir rüber wollten und [...] sagen: Wir wollen etwas wissen und du mußt die Wahrheit sagen, hörst du? [...] Vielleicht wird der Beamte ihm drohen, vielleicht an seinen Arm reißen, damit Aleksej nicht vergißt, wieviel stärker einer wie er war. [...] Was wil eure Mutter drüben? Liebt sie den Mann? Habt ihr gesehen, ob er sie küßt? [...] Es gab nur falsche Antworten. [...] Falsche Antworten. Nicht einmal das wußte Aleksej." (Frank 2003, 11)

Nach den Kindern wird auch die Hauptfigur verhört. 18 Seiten lang wird es ausführlich erzählt. Sie wird abwechselnd von unterschiedlichen Beamten nacheinander befragt und zweimal auch physisch untersucht, sogar gynäkologisch. Das ist erniedrigend, aber Nelly ist sich der Methoden bewusst und darauf gefasst. Inzwischen denkt sie: „Sie wollen dich ärgern, dachte ich, nichts Böses, sie wollen dich ärgern. Welche Genugtuung für diesen kleinen großen Beamten wohl in solchen Fragen und Antworten steckte?" (Frank 2003, 17). Manchmal reagiert Nelly mit einer Phrase „das wissen Sie”, und damit wird bestätigt, dass diese Befragung wirklich einen psychischen Druck bewirken soll. Sie muss sich vor männlichen Beamten ausziehen. Die körperliche Nacktheit Nellys steht im Gegensatz zu den uniformiert bekleideten Beamten. Sie „hätte unbemerkt vor Scham im Boden versinken können”. „Ihm schien meine Nacktheit peinlicher als mir" (Frank 2003, 27). So denkt Nelly in dieser Situation der höchsten Auslieferung und Erniedrigung. Bei der Untersuchung setzt sich Nelly mit den Gedanken des Kalten Krieges auseinander. Das Regime und wie das Regime mit seinen Bürgern umgeht, ist eine Reaktion auf den Kalten Krieg. Diese Scham und Inhumanität signalisiert sehr gut die Nachtmetapher, die der verzweifelten, bitteren und zugleich resignierten Frage von Nelly zu entnehmen ist: „Wie schwarz sollte die Nacht auch geworden sein?” (Frank 2003, 18). Schwarz steht für diese Schande und Unwürde.

„Nur hatten wir keinen Krieg. Zumindest hatte ich das Wort bislang nicht so geglaubt. Auch mit dem Zusatz kalt nicht. Was hieß das schon kalt? [...] unter Krieg stellte ich mir das andere vor, das, was ich nicht erfahren hatte. [...] Daß wir im Krieg lebten, hatte ich bislang nicht so recht bemerkt. Ich schämte mich dafür. Aber es war sicher nicht die Scham, nach der da in mir gesucht wurde." (Frank 2003, 30)

Und da wird sie von bitteren Erfahrungen des Kalten Krieges überflutet. Dass z. B. auch ihre 87 jährige Großmutter vor drei Jahren nach zahlreichen legitimen Grenzübergängen untersucht wurde, um ihr unter einem ähnlichen Vorwand [wie Nelly jetzt], sämtliche Kronen und Brücken zu entnehmen. „Nur hofften sie bei ihr wohl, Material eines westlichen Geheimdienstes zu entdecken" (Frank 2003, 30). Der Hauptfigur bleibt als Mensch nur der hilflose Zynismus übrig, das Wesen des kommunistischen Regimes zu entlarven. Typisch ist für die Befragungen, dass die Beamten den Infinitiv als Befehlgebung benutzen, wie „setzen”, „abtreten”. Nach 
dieser Misere in der DDR wird die Befragungsprozedur in der BRD fortgesetzt. Die Neuankömmlinge aus dem Ostblock erfahren, keine Unabhängigkeit und Freiheit, sondern nur eine andere Art von Abhängigkeit und Unfreiheit. (Brussig 2004) Auf die Bemerkung des amerikanischen Beamten, dass Nelly bei innen in Sicherheit sei, antwortet Nelly, dass sie so unterrichtet sei, dass man am sichersten in einem kommunistischen Land mit einer Mauer sein kann. (Frank 2003, 62) Und sie erzählt, dass sie schon der Fragerei müde sei:

„Sehen Sie, ein halbes Leben lang hat mich die Staatssicherheit befragt, heute sind Sie dran, morgen die Briten, und übermorgen wollen die Franzosen. Wann darf der bundesdeutsche Geheimdienst dran? Und an der Grenze haben mich unsere Staatsdiener [...] befragt. Mein Kopf ist leer. [...] ununterbrochen werde ich verhört." (Frank 2003, 74)

Trotzdem wird sie auch hier mehrmals verhört, hauptsächlich wegen ihres Geliebten, Wassilij, von dem die Amerikaner vermuten, dass er ein Doppelagent war. Nelly als Staatsbürger ist nur ein Spielball zwischen den Großmächten, überall wird sie in ihrer Menschenwürde und Freiheit verletzt, nur die Art und Weise der Großmächte unterscheiden sich, wie sie mit den Menschen umgehen.

\section{Motivkomplexe im Film}

Der Grenzübergang wird im Film etwas schneller inszeniert als im Buch, aber die wichtigsten Motive korrespondieren mit denen des Romans. Das Gebäude, in dem Nelly verhört und untersucht wird, wird nicht so ausführlich erfasst. Im Film hat sie nur ein Kind, Aleksej und er geht nur auf die Toilette, er wird nicht verhört. Nellys Angst um ihren Sohn und die vielen inneren Monologe werden nicht wiedergegeben. Beim Grenzübergang ist aber die schauspielerische Leistung hervorragend, die in der Mimik zum Ausdruck kommt. Auf den Bildern des Filmes ist der gleiche Ort, die gleiche Zeit, mit 30 Sekunden Differenz erfasst.

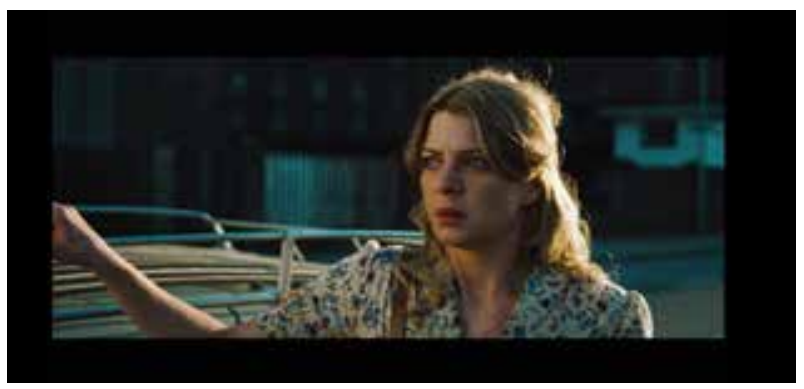

Zuerst (im Film bei 8:15-8:45) blickt Nelly in die DDR zurück, sie ist schon über die Brücke gefahren und bleibt dort stehen. Der Schreck, der Abscheu und ein düsterer Blick ist auf dem Gesicht zu entnehmen. 


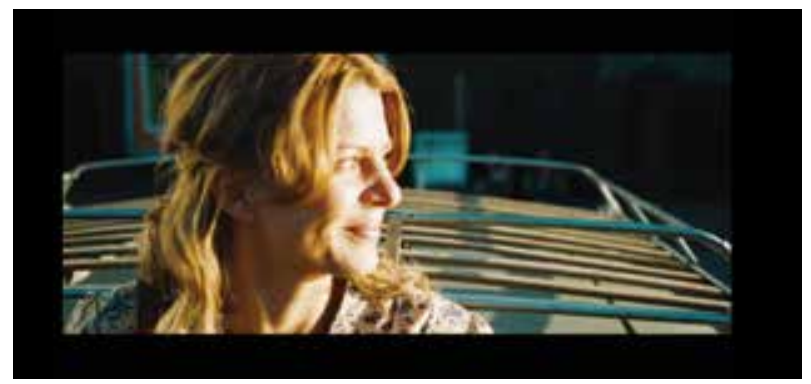

Auf dem anderen Bild (im Film bei 9:00-9:10) blickt sie nach vorne, ihr gegenüber scheint noch die untergehende Sonne, sie beleuchtet ihre Haare und Gesicht. Es ist ein Bild von Optimismus, Hoffnung und Freude. Zwei Seiten der Brücke, der Grenze, zwei Pole des Lebens, die Vergangenheit und die Zukunft, die Brücke symbolisiert das Jetzt, das Dazwischen, in dem sich Nelly befindet.

Bei der Ankunft in Marienfelde sieht man einen Gebäudekomplex, der mit einem Betonzaun umgeben ist. Die Flüchtlinge müssen durch ein Tor gehen. Die Begrenzung als Motiv ist auch hier erfasst.

Ihr letztes Verhör in der DDR wird im Film inszeniert. Die Hauptfigur wird beim Grenzübergang abgeführt. Sie wartet allein, und als der Beamte kommt, blickt Nelly auf inn. Dieser Blick und dass sie sitzt, der Mann aber steht, zeigt ihre untergeordnete und ausgelieferte Position. Nelly beherrscht sich die ganze Zeit, sie ist mutig und entschlossen - auch bei den größten Demütigungen.

Die Verhöre in der DDR und in der BRD belasten sie sehr. In der DDR ist die psychische auch mit einer physischen Belastung verbunden, was ihr Schmerzen verursacht. In der BRD wird Nelly aber mit der amerikanischen Staatssicherheit konfrontiert und erkennt den Grund ihrer Verhöre: Wassilij ist es, ihr Lebensgefährte, der sie in diese Bedrohung brachte. Sie hat diesen Mann geliebt, ihn verloren und jetzt möchte sie neuanfangen. Aber die amerikanischen Offiziere erschrecken sie damit, dass der Tod von Wassilij eine Täuschung gewesen sein könnte. Das erschüttert Nelly und ihre Angst wird auch in der BRD neu erweckt. Nelly schwankt in ihren Gefühlen zu Wassilij, sie liebt ihn, sie vermisst ihn, aber sie hat nun Angst. In einer Szene hat sie eine Vorstellung, einen Tagtraum, in dem sie Wassilij umarmt. Sie sucht nach Sicherheit und Trost, aber das hat sie nur in ihren Gedanken und ihren Wünschen. Die Filmkritik bringt es auf den Punkt, indem sie behauptet, „zwischen den ideologischen Fronten hat sie eine bedrückende Erkenntnis: wie ähnlich sich beide Systeme doch anfühlen” (Buß 2014). Die zwei Systeme ähneln sich sonderbar, in beiden Welten ist Nelly Grenzen ausgesetzt. 


\section{Fazit}

Man kann beobachten, dass bestimmte Motive und Metaphern dominanter sind oder wiederholt vorkommen. Daraus ergibt sich die Symbolik des Buches. Typisch sind die schwarz-weiß Kontraste, die Vorstellung vom unterirdischen Labyrinth, in dem Menschen verschwinden, die Brücke, der Zaun, die Mauer, die Uniform und das Feuer. Das Anfangsbild von der untergehenden Sonne mit Feuerstreifen findet in dem Schlussbild seine Vollendung. Die Geschichte endet zu Weihnachten im Notaufnahmelager. Den Flüchtlingskindern werden ein Weihnachtsbaum und Geschenke gespendet, es wird die Ode An die Freude von Schiller gesungen und der Weihnachtsbaum beginnt zu brennen. Mit diesem Bild des Feuers endet der Roman, so fungiert das Feuer als Rahmen. Nellys Weg ist im Buch ein Kreis. Von der Begrenzung kommt sie über die Entgrenzung wieder zur Begrenzung, zum brennenden Weihnachtsbaum. Im Film findet man den gleichen Kreis, allerdings tritt Nelly am Ende aus dieser Begrenzung hinaus, indem sie eine Wohnung mietet und das Notaufnahmelager verlässt.

Aber die Motive der Verhöre und Nellys Ausgeliefertsein in beiden Welten zeigen dem Zuschauer, „wie jedes System, ob demokratisch oder autoritär, den Menschen seinen Stempel aufzudrücken versucht. Das Unpolitische kann es nicht geben, wenn alles um einen herum Politik ist." (Buß 2014)

\section{Literaturverzeichnis}

Brussig, Thomas (2004): Unsanfte Landung. https://www.spiegel.de/spiegel/ print/d-28721244.html [Abgerufem am: 10.02.2020].

Buß, Christian (2014): Furioses DDR-Fluchtdrama „Westen”. In der Twilight Zone des Kalten Krieges.

https://www.spiegel.de/kultur/kino/westen-kinodrama-mit-joerdis-triebel-von-christian-schwochow-a-959242.html Abgerufem am: 10.02.2020

Franck, Julia (2003): Lagerfeuer. Köln: DuMont Verlag.

Schmelcher, Antje (2006): Julia Franck: Narben sind häufig taub. https://www.welt.de/ print-welt/article256146/Narben-sind-haeufig-taub.html, Abgerufen am 10.02.2020

Schwochow, Christian (2013): Westen. DVD. 